\title{
MAGNETIC SPECTRAL BOUNDS ON STARLIKE PLANE DOMAINS
}

\author{
R.S. LAugesen ${ }^{1}$ And B.A. Siudeja ${ }^{2}$
}

\begin{abstract}
We develop sharp upper bounds for energy levels of the magnetic Laplacian on starlike plane domains, under either Dirichlet or Neumann boundary conditions and assuming a constant magnetic field in the transverse direction. Our main result says that $\sum_{j=1}^{n} \Phi\left(\lambda_{j} A / G\right)$ is maximal for a disk whenever $\Phi$ is concave increasing, $n \geq 1$, the domain has area $A$, and $\lambda_{j}$ is the $j$ th Dirichlet eigenvalue of the magnetic Laplacian $\left(i \nabla+\frac{\beta}{2 A}\left(-x_{2}, x_{1}\right)\right)^{2}$. Here the flux $\beta$ is constant, and the scale invariant factor $G$ penalizes deviations from roundness, meaning $G \geq 1$ for all domains and $G=1$ for disks.
\end{abstract}

Mathematics Subject Classification. 35P15, 35J20.

Received January 5, 2014. Revised June 3, 2014.

Published online May 13, 2015.

\section{INTRODUCTION}

\subsection{Overview}

The energy levels of a charged quantum particle are difficult to understand analytically. We aim for insight into the behavior of these energy levels on a two dimensional region by proving they are maximal for a disk, when normalized by a certain geometric factor depending on the boundary shape of the original confinement region.

Specifically, we develop sharp upper bounds for energy levels of the magnetic Laplacian on starlike plane domains, under either Dirichlet or Neumann boundary conditions, assuming a constant magnetic field in the transverse direction. The spectral functionals we consider include the ground state energy, sums and products of energy levels, the spectral zeta function, and the partition function.

For the special case of the ground state energy, our upper bound complements a lower bound of Faber-Krahn type due to Erdös [6], which says that the first eigenvalue $\lambda_{1}$ of the magnetic Dirichlet Laplacian is minimal for the disk of the same area. Combining these upper and lower bounds gives a pair of inequalities:

$$
1 \leq \frac{\lambda_{1}(\Omega)}{\lambda_{1}\left(\Omega^{*}\right)} \leq G(\Omega)
$$

when $\Omega \subset \mathbb{R}^{2}$ is a starlike plane domain, $\Omega^{*}$ is the disk of the same area, and the computable geometric factor $G(\Omega)$ measures how far the domain is from being circular (with $G=1$ for a disk; see the definition in the

\footnotetext{
Keywords and phrases. Isoperimetric, spectral zeta, heat trace, partition function, Pauli operator.

1 Department of Mathematics, University of Illinois, Urbana, IL 61801, USA. Laugesen@illinois.edu

2 Department of Mathematics, University of Oregon, Eugene, OR 97403, USA. Siudeja@uoregon.edu
} 
next section). Note that the upper estimate in this paper requires starlikeness of the domain, whereas the lower estimate due to Erdös holds whenever the domain is merely bounded. The upper estimate has the advantage, though, of applying under Neumann boundary conditions too.

Our results apply to a huge class of spectral functionals beyond the ground state energy. Theorem 2.1 shows that

$$
\sum_{j=1}^{n} \Phi\left(\frac{\lambda_{j}(\Omega)}{G(\Omega)}\right) \leq \sum_{j=1}^{n} \Phi\left(\lambda_{j}\left(\Omega^{*}\right)\right)
$$

whenever $n \geq 1$ and $\Phi: \mathbb{R}_{+} \rightarrow \mathbb{R}$ is concave and increasing, and $\lambda_{j}$ is the $j$ th eigenvalue of the magnetic Dirichlet Laplacian

\subsection{Formulating the problem}

Imagine a uniform, vertical magnetic field passing through a cylinder $\Omega \times \mathbb{R}$ in which a charged, spinless quantum particle is confined. The particle moves freely in the vertical direction, and so its wavefunction can be written as a plane wave in the $x_{3}$-variable multiplied by a wavefunction of the horizontal coordinates $x_{1}, x_{2}$. We aim to study the quantized energy levels of this horizontal motion, which are eigenvalues of a Schrödinger operator known as the magnetic Laplacian; see for example Erdös [7], Helffer et al. $[9,12]$.

To formulate the problem mathematically, consider a bounded plane domain $\Omega$ with area $A$, and fix $\beta \geq 0$. The magnetic Laplacian on $\Omega$ is the symmetric operator

$$
(i \nabla+F)^{2}
$$

where the vector potential

$$
F(x)=\frac{\beta}{2 A}\left(-x_{2}, x_{1}\right)
$$

is chosen to generate a transverse magnetic field $\nabla \times F=(0,0, \beta / A)$ of strength $B=\beta / A$. The constant $\beta$ represents the magnetic flux through the domain. The magnetic Laplacian reduces to the usual Laplacian when $\beta=0$, because then $F \equiv 0$.

The magnetic Laplacian has discrete spectrum, assuming Dirichlet boundary conditions on $\partial \Omega$, with eigenvalues $\left\{\lambda_{j}\right\}$ satisfying

$$
0<\lambda_{1} \leq \lambda_{2} \leq \lambda_{3} \leq \ldots
$$

Gauge invariance guarantees that these eigenvalues are unchanged if we replace the vector potential $F$ with any other vector field having the same curl (and thus generating the same magnetic field). For a leisurely treatment of this and other invariance properties of the spectrum of the magnetic Laplacian (see [17], Sect. 2 and Appendix A). For the purposes of this paper, the "rotational" vector potential (1.1) is a convenient choice.

We denote by $\left\{u_{j}\right\}$ a corresponding sequence of $L^{2}$-orthonormal eigenfunctions, with

$$
\left\{\begin{array}{r}
(i \nabla+F)^{2} u_{j}=\lambda_{j} u_{j} \text { in } \Omega, \\
u_{j}=0 \text { on } \partial \Omega .
\end{array}\right.
$$

The magnetic Laplacian $(i \nabla+F)^{2}$ on the left side of the equation scales like $1 /(\text { length })^{2}$, and hence the eigenvalues scale the same way. Thus the area-normalized eigenvalue $\lambda_{j} A$ is invariant under scaling of the domain.

Assume $\Omega$ is a Lipschitz-starlike plane domain, by which we mean

$$
\Omega=\left\{r \mathrm{e}^{\mathrm{i} \theta}: 0 \leq r<R(\theta)\right\}
$$

where the radius function $R(\cdot)$ is positive, $2 \pi$-periodic, and Lipschitz continuous. Just as in our earlier work on the Laplacian [18], Section 10, we define two scale-invariant geometric factors in terms of the radius function, 


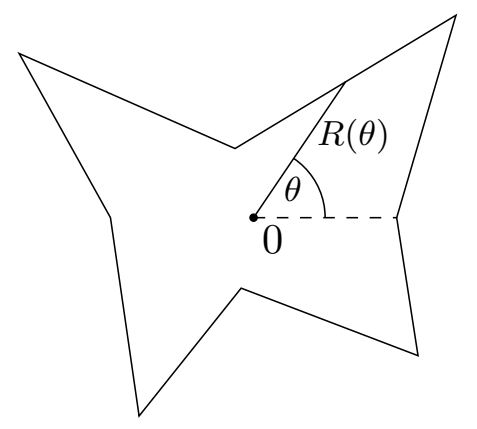

Figure 1. A starlike domain with radius function $R(\theta)$.

by

$$
\begin{aligned}
& G_{0}=1+\frac{1}{2 \pi} \int_{0}^{2 \pi}(\log R)^{\prime}(\theta)^{2} \mathrm{~d} \theta \\
& G_{1}=\frac{\frac{1}{2 \pi} \int_{0}^{2 \pi} R(\theta)^{4} \mathrm{~d} \theta}{\left(\frac{1}{2 \pi} \int_{0}^{2 \pi} R(\theta)^{2} \mathrm{~d} \theta\right)^{2}}=\frac{2 \pi I_{\text {origin }}}{A^{2}},
\end{aligned}
$$

where $I_{\text {origin }}=\int_{\Omega}|x|^{2} \mathrm{~d} x$ is the polar moment of inertia of $\Omega$ about the origin. Obviously

$$
G_{0} \geq 1 \quad \text { and } \quad G_{1} \geq 1
$$

with equality if and only if the domain is a disk centered at the origin ( $R \equiv$ const.).

Take the maximum of the two geometric factors, and call it $G$ :

$$
G=\max \left\{G_{0}, G_{1}\right\} \geq 1 .
$$

We interpret $G$ as measuring the deviation of the domain from roundness. Deviation can occur in two ways: an oscillatory boundary would make $R^{\prime}$ large and hence $G_{0}$ large, whereas an elongated boundary (such as an eccentric ellipse) would force $R^{4}$ to vary more than $R^{2}$ and hence would make $G_{1}$ large. Calculations are generally required in order to determine which of $G_{0}$ or $G_{1}$ is larger (see [18], Sect. 10).

\section{MAin RESUlts}

\subsection{Dirichlet boundary conditions}

Our main result says that the disk maximizes eigenvalues of the magnetic Laplacian under suitable geometric scaling normalized by area and $G$.

Theorem 2.1 (Dirichlet magnetic Laplacian). Suppose $\Omega=\left\{r \mathrm{e}^{\mathrm{i} \theta}: 0 \leq r<R(\theta)\right\}$ is a Lipschitz-starlike plane domain. Fix $\beta \geq 0$ and $n \geq 1$.

If $\Phi: \mathbb{R}_{+} \rightarrow \mathbb{R}$ is concave and increasing then $\sum_{j=1}^{n} \Phi\left(\lambda_{j} A / G\right)$ is maximal when $\Omega$ is a disk centered at the origin.

When $\Phi$ is the identify function, the theorem maximizes the sum $\lambda_{1}+\ldots+\lambda_{n}$, which represents the energy for filling the first $n$ quantum states when at most one particle can occupy each state (the Pauli exclusion principle). This special case is central to proving the theorem, as we will see in Section 5. 


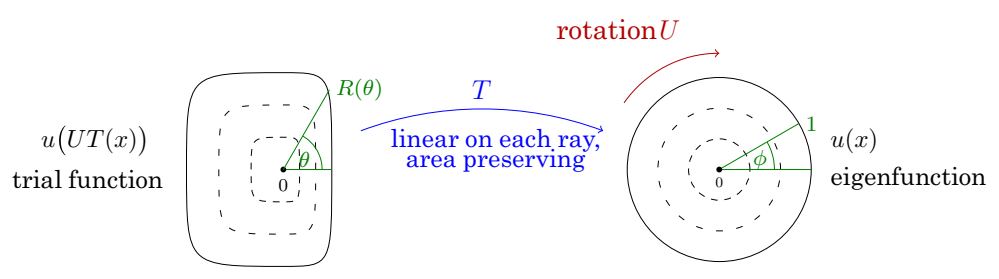

Figure 2. A linear-on-rays transformation from a domain $\Omega$ of area $\pi$ to the unit disk. To insure that the mapping preserves area locally, we require $R(\theta)^{2} \mathrm{~d} \theta=d \phi$.

As a consequence of the theorem, we extremize several interesting spectral functionals:

Corollary 2.2. Fix $\beta \geq 0$ and $n \geq 1$. Each of the following scale invariant functionals achieves its maximum value when the Lipschitz-starlike domain $\Omega$ is a centered disk:

$$
\lambda_{1} A / G, \quad\left(\lambda_{1}^{s}+\ldots+\lambda_{n}^{s}\right)^{1 / s} A / G, \quad \sqrt[n]{\lambda_{1} \lambda_{2} \ldots, \lambda_{n}} A / G,
$$

for each exponent $0<s \leq 1$.

Equality statement: if $\lambda_{1} A /\left.G\right|_{\Omega}=\lambda_{1} A /\left.G\right|_{\text {disk }}$ then $\Omega$ is a centered disk.

Furthermore, each partial sum of the spectral zeta function and of the trace of the heat kernel is minimal when $\Omega$ is a centered disk. That is, the functionals

$$
\sum_{j=1}^{n}\left(\lambda_{j} A / G\right)^{s} \quad \text { and } \quad \sum_{j=1}^{n} \exp \left(-\lambda_{j} A t / G\right)
$$

attain their smallest value when $\Omega$ is a centered disk, for each $s<0<t$.

The eigenvalues for the disk, which is the extremal domain in the Corollary, can be computed in terms of zeros of certain Kummer functions. We will not need that fact in our proof of the theorem or corollary, but readers who are interested may consult the detailed treatment by Son ([23], Chap. 3), which includes informative plots of the eigenvalues as functions of the flux $\beta$.

Theorem 2.1 can be strengthened by replacing $G$, which is the maximum of $G_{0}$ and $G_{1}$, with certain convex combinations of $G_{0}$ and $G_{1}$. Further improvements involve choosing a "good" location for the origin so as to reduce the values of $G_{0}$ and $G_{1}$. See our discussion in the case of zero magnetic field ([18], Sects. 9 and 10).

We prove Theorem 2.1 by adapting our method from the non-magnetic case ([18], Thm. 1.1). Just as in that earlier work, we transform $\Omega$ into a disk while controlling angular information in the relevant Rayleigh quotient. The transformation is linear on rays and has constant Jacobian, as indicated in Figure 2. Note that wherever the transformation stretches radially it must compress angularly, in order to preserve area. This constant Jacobian condition guarantees that when we transplant orthogonal eigenfunctions from the disk we will obtain orthogonal trial functions on $\Omega$.

One cannot know in general which orientation of the trial function in $\Omega$ will yield the smallest value for the Rayleigh quotient. The optimal orientation typically differs for each index $j$, in any case, and so we aim instead for the average case - we consider all possible orientations of the trial function, by employing the arbitrary rotation $U$ of the disk in Figure 2.

\subsection{Perturbations of the disk}

Let us apply the theorem to the ground state energy of a nearly circular domain. Suppose $P(\theta)$ is a Lipschitz continuous, $2 \pi$-periodic function with Fourier series

$$
P(\theta)=\sum_{n \in \mathbb{Z}} p_{n} \mathrm{e}^{\mathrm{i} n \theta}
$$


where $p_{-n}=\overline{p_{n}}$ since $P$ is real-valued. Define a plane domain $\Omega_{\varepsilon}=\left\{r \mathrm{e}^{\mathrm{i} \theta}: 0 \leq r<1+\varepsilon P(\theta)\right\}$, and assume $\varepsilon$ is small enough that the radius $1+\varepsilon P(\theta)$ is positive for all $\theta$. Obviously $\Omega_{\varepsilon}$ is a perturbation of the unit disk, when $\varepsilon$ is small. Write

$$
\lambda_{\varepsilon}=\lambda_{1}\left(\Omega_{\varepsilon}\right)
$$

for the first Dirichlet eigenvalue of the magnetic Laplacian on $\Omega_{\varepsilon}$. Let $A_{\varepsilon}$ be the area of the domain, and remember that the flux through each domain $\Omega_{\varepsilon}$ is the same, namely $\beta$. Denote the unit disk centered at the origin by $\mathbb{D}$.

Corollary 2.3 (Nearly circular domains). The first magnetic eigenvalue of the domain $\Omega_{\varepsilon}$ is bounded above and below in terms of the boundary perturbation, with

$$
1 \leq \frac{\lambda_{\varepsilon} A_{\varepsilon}}{\lambda_{1}(\mathbb{D}) \pi} \leq 1+2 \varepsilon^{2} \max \left\{\sum_{n=1}^{\infty} n^{2}\left|p_{n}\right|^{2}, 4 \sum_{n=1}^{\infty}\left|p_{n}\right|^{2}\right\}+O\left(\varepsilon^{3}\right)=G\left(\Omega_{\varepsilon}\right)
$$

as $\varepsilon \rightarrow 0$ with $P$ fixed.

The lower bound in the corollary is the Faber-Krahn type inequality due to Erdös [6]. The upper bound follows from Theorem 2.1 with $n=1$, as we show in Section 6 .

Next we carry out a formal perturbation analysis on the ground state energy of a nearly circular domain, in order to investigate whether the estimate in the corollary is best possible. We will adapt to the magnetic case a method developed for the Laplacian (the case $\beta=0$ ) by Rayleigh ([24], Sect. 210) and simplified by Pólya and Szegö ([21], pp. 132-134). The resulting asymptotic formula (2.1) below should be understood as "the expression resulting from a formal analysis" - we do not claim that it holds in the literal sense. Note the corresponding asymptotic formula for the Laplacian can be proved rigorously by Hadamard-type boundary variation techniques (see Henry's treatment [14], p. 35), but the effort required for a rigorous proof seems out of proportion to the reward, in the magnetic case.

In order to state the formal perturbation result, write

$$
M(a, b, z)=1+\frac{a}{b} z+\frac{a(a+1)}{b(b+1)} \frac{z^{2}}{2 !}+\ldots
$$

for the Kummer function ([20], Chap. 13), which is known also as the confluent hypergeometric function. Denote its $z$-derivative by $M^{\prime}$. Assume $p_{0}=0$, which is harmless since it essentially amounts to a rescaling of the perturbed domain. Fix $\beta>0$ and let

$$
a_{0}=a_{0}(\beta)=\frac{1}{2}\left(1-\lambda_{0} \pi / \beta\right) .
$$

Then perturbation analysis yields an asymptotic approximation to $\lambda_{\varepsilon} A_{\varepsilon}$ as $\varepsilon \rightarrow 0$ (with $P$ fixed):

$$
\lambda_{\varepsilon} A_{\varepsilon}=\lambda_{1}(\mathbb{D}) \pi+\left(\sum_{n=2}^{\infty} c\left|p_{n}\right|^{2} q_{n}\right) \varepsilon^{2}+O\left(\varepsilon^{3}\right)
$$

where

$$
\begin{aligned}
z & =\frac{\beta}{2 \pi}, \\
c & =\frac{-M^{\prime}\left(a_{0}, 1, z\right)}{\frac{\partial M}{\partial a}\left(a_{0}, 1, z\right)} \frac{4 \beta^{2}}{\pi}, \\
q_{n} & =1+n-z+z(\log M)^{\prime}\left(a_{0}, n+1, z\right)+z(\log M)^{\prime}\left(a_{0}+n, n+1, z\right) .
\end{aligned}
$$


We establish (2.1) in Section 6, and show there that $q_{n}=n+O(1)$ as $n \rightarrow \infty$. Thus the $\varepsilon^{2}$-term in the formal asymptotic series (2.1) involves the $H^{1 / 2}$-norm of the boundary perturbation, while the second order term in Corollary 2.3 is essentially the $H^{1}$-norm. Hence the asymptotic formula is in that sense sharper, although on the other hand we have proved it only formally and so have no control over its error term. It is an open problem to prove an inequality (or error estimate) that holds for each $\varepsilon>0$ and which captures the asymptotic series to second order as $\varepsilon \rightarrow 0$. This problem is open even for the Laplacian.

Remark 2.4. The summation in (2.1) begins with $n=2$, which leads one to ask: might the first eigenvalue actually decrease under boundary perturbations of type $n=1$, that is, $\cos \theta$ or $\sin \theta$ perturbations? No! The ground state energy increases under such perturbations, as follows from the magnetic Faber-Krahn result of Erdös (the lower bound in Cor. 2.3). This observation highlights the subtlety of Erdös's result, and of the original Faber-Krahn theorem in the nonmagnetic case.

\subsection{Neumann boundary conditions}

Assume the magnetic field is nonzero in what follows, meaning $\beta>0$. (The zero field case was treated in the earlier paper [18]). Write $\left\{\mu_{j}\right\}$ for the Neumann eigenvalues of the magnetic Laplacian on $\Omega$, so that the corresponding $L^{2}$-orthonormal eigenfunctions $u_{j}$ satisfy

$$
\left\{\begin{array}{l}
(i \nabla+F)^{2} u_{j}=\mu_{j} u_{j} \text { in } \Omega \\
\boldsymbol{n} \cdot(i \nabla+F) u=0 \text { on } \partial \Omega
\end{array}\right.
$$

where as before, $F(x)=\frac{\beta}{2 A}\left(-x_{2}, x_{1}\right)$. The boundary condition arises naturally from minimization of the Rayleigh quotient, and it plays no role in our proofs. The eigenvalues satisfy

$$
0<\mu_{1} \leq \mu_{2} \leq \mu_{3} \leq \ldots
$$

where we note that positivity of the first eigenvalue holds because the field is nonzero (see, for example, [17], Lem. A.8).

Theorem 2.5 (Neumann magnetic Laplacian). Assume $\beta>0$. Then Theorem 2.1 holds with the Dirichlet eigenvalue $\lambda_{j}$ replaced by the Neumann eigenvalue $\mu_{j}$.

The proof mimics the Dirichlet case. The needed changes are indicated in Section 5.

The Neumann magnetic eigenvalues on a disk can in principle be computed in terms of Kummer functions, although in practice the equations become rather complicated. The eigenvalue branches display fascinating behavior. For example, a numerical study by Saint-James [22] reveals that the Neumann ground state is nonradial when the flux $\beta$ is large enough; indeed, the ground state appears to have angular dependence $\mathrm{e}^{\mathrm{i} \theta n(\beta)}$ for some positive integer $n(\beta)$ that increases to infinity as $\beta$ increases to infinity. Incidentally, this non-radial property of the Neumann ground state means that our proof of the equality statement for the first Dirichlet eigenvalue in Corollary 2.2 does not extend to the Neumann case.

Despite these complications, Helffer and Morame [12] were able to prove monotonicity of the lowest Neumann eigenvalue of the disk for large values of $\beta$ (corresponding to small semi-classical constants). For domains with corners, oscillatory behavior of the low eigenfunctions was observed both numerically and theoretically by Bonnaillie-Noël et al. [5], again for large values of $\beta$. See the references in that paper for other semi-classical results.

\subsection{Relevant literature, and the contributions of this paper}

Few isoperimetric type inequalities are known for magnetic eigenvalues. This paucity stands in stark contrast to the rich body of work developed for the nonmagnetic Laplacian over the past century, for which one may consult the surveys by Ashbaugh and Benguria [2] or Benguria and Linde [4], and the monographs of Bandle [3], 
Henrot [13], Kesavan [15] and Pólya-Szegö [21]. The main contribution of this paper is to prove the first known sharp upper bounds for magnetic spectral functionals on more-or-less general plane domains.

This paper generalizes our earlier work on eigenvalues of the Laplacian [18], that is, on the case of zero magnetic field. Specifically, Theorem 2.1 in this paper is the magnetic analogue of Theorem 1.1 from that earlier work. (The result for $\lambda_{1}$ is slightly stronger in that earlier work, because when $\beta=0$ one finds $\alpha_{1}=0$ in (5.5) below, and hence one may use $G_{0}$ instead of $G$ on the first eigenvalue.) Those earlier results hold in all dimensions [18], Theorem 3.1, with balls as the maximizers. We restrict in this paper to two dimensions, though, because in three dimensions and higher, introducing a magnetic field creates a preferred direction in the problem, which breaks the symmetry and thus renders our proof invalid.

It is much simpler to work in the plane than in higher dimensions. The proof of our main result, Theorem 2.1, relies on the special fact that rotations commute in two dimensions: we exploit this fact to construct a proof that is both shorter and easier to understand than in our earlier work on the Laplacian. Thus for readers who are new to this subject, we recommend beginning with the proof of Theorem 2.1 in the zero-field case, taking $\beta=0$ throughout the proof, and only then turning to the magnetic case $(\beta>0)$ or to the higher dimensional case in our earlier paper [18].

Another work to which this current paper owes a debt is that of Laugesen, Liang and Roy [17]. They treated a restricted class of domains, namely linear images of rotationally symmetric domains such as regular polygons, and obtained sharp upper bounds on magnetic eigenvalue sums with the maximizing domains being the original rotationally symmetric domains. For example, the centered equilateral triangle was shown to maximize the eigenvalue sum $\left(\lambda_{1}+\ldots+\lambda_{n}\right) A / G_{1}$ among all triangles. (The authors could have subsequently invoked majorization to pass to spectral functionals such as the partition function, like in this paper, but did not do so.)

One difference between the work of Laugesen et al. and the current paper is that here we average over the full group of rotations instead of over discrete subgroups such as the 3-fold rotations for the equilateral triangle. Thus we avoid the tight frame theory that was needed in the earlier paper [17]. Another difference is that the transformations in that paper were rather simple (in fact, globally linear), whereas in the current paper we must use more complicated linear-on-rays transformations such as shown in Figure 2, in order to map the disk to general starlike domains. This additional complexity forces the inclusion of the boundary oscillation factor $G_{0}$ in our theorems; it was not needed in the earlier work, since a linear transformation stretches without oscillation.

Lastly we mention some "asymptotically sharp" inequalties, for which equality holds for every domain in the limit $n \rightarrow \infty$. (In contrast, the results in this paper can be called "geometrically sharp" because a single extremal domain exists for each fixed $n$.) An inequality of Berezin-Li-Yau type holds for sums of magnetic eigenvalues, by work of Erdös et al. [8], extending results of Laptev and Weidl [16]. See also a later work of Frank, Laptev and Molchanov [10]. On the other hand, the magnetic Pólya conjecture, which would claim that the Weyl asymptotic formula provides a lower bound for each individual magnetic eigenvalue, was disproved by Frank et al. [11] by constructing a counterexample from square domains.

\subsection{Open problems}

Erdös proved under Dirichlet boundary conditions that the magnetic ground state energy is minimal for a disk of the same area [6]. In scale invariant terms, he proved $\lambda_{1} A$ is minimal for the disk. This result suggests several open problems.

Is the scale invariant magnetic partition function $\sum_{j=1}^{\infty} \mathrm{e}^{-\lambda_{j} A t}$ maximal for the disk, for each $t>0$ ? Luttinger [19] proved the result for the Laplacian $(\beta=0)$. Note that letting $t \rightarrow \infty$ would recover the minimality of the first eigenvalue.

Next, for the Neumann spectrum does one have minimality of $\mu_{1} A$ for the disk? This conjecture holds trivially for the Laplace operator, because there $\mu_{1}=0$ for all domains. Thus one should begin by investigating the conjecture for weak magnetic fields (small $\beta$-values) by means of a perturbation analysis. Even if the conjecture holds for weak fields, it might fail for strong fields because the nature of the ground state changes: the Neumann ground state of the disk is radial for small values of $\beta$ but has angular dependence when $\beta$ is large (see comments above). 


\section{Results For the Pauli operator}

To study a charged particle with spin $1 / 2$, we investigate the energy levels of the Pauli operator $H_{P}=$ $(\sigma \cdot(i \nabla+F))^{2}$ (see [7], for example). Here $\sigma=\left(\sigma_{1}, \sigma_{2}, \sigma_{3}\right)$ is the 3-tuple of self-adjoint Pauli matrices:

$$
\sigma_{1}=\left(\begin{array}{cc}
0 & 1 \\
1 & 0
\end{array}\right), \quad \sigma_{2}=\left(\begin{array}{cc}
0 & -i \\
i & 0
\end{array}\right), \quad \sigma_{3}=\left(\begin{array}{cc}
1 & 0 \\
0 & -1
\end{array}\right)
$$

The Pauli operator acts on spinors, that is, on 2-component complex vector fields of the form $\psi_{-}=\left(\begin{array}{l}\psi_{+} \\ \psi_{-}\end{array}\right)$. For planar motion with a perpendicular magnetic field $(0,0, \beta / A)$, we may assume the wavefunction $\psi$ is independent of $x_{3}$ and that the gradient $\nabla=\left(\partial_{1}, \partial_{2}\right)$ and vector potential $F=\left(F_{1}, F_{2}\right)=\left(-x_{2}, x_{1}\right) \beta / 2 A$ have only two components. Thus the planar Pauli operator is the formally self-adjoint operator

$$
H_{P}=\left(\sum_{m=1}^{2} \sigma_{m}\left(i \partial_{m}+F_{m}\right)\right)^{2}
$$

with corresponding Rayleigh quotient

$$
\operatorname{Ray}_{P}[\psi]=\frac{\int_{\Omega}\left|\sum_{m=1}^{2} \sigma_{m}\left(i \partial_{m}+F_{m}\right) \psi\right|^{2} \mathrm{~d} x}{\int_{\Omega}|\psi|^{2} \mathrm{~d} x} .
$$

The numerator is known to be elliptic for $\psi \in H_{0}^{1}\left(\Omega ; \mathbb{C}^{2}\right)$, meaning it is bounded below by a constant times $\int_{\Omega}|\nabla \psi|^{2} \mathrm{~d} x$ minus a constant times $\int_{\Omega}|\psi|^{2} \mathrm{~d} x$. (A brief demonstration of ellipticity is included in Sect. 7). Hence the Dirichlet spectrum of the Pauli operator is discrete, by the spectral theorem for quadratic forms. We place the eigenvalues in increasing order, so that

$$
0<\lambda_{1}^{P} \leq \lambda_{2}^{P} \leq \lambda_{3}^{P} \leq \ldots
$$

where " $P$ " stands for Pauli. Positivity of the first eigenvalue will be justified in Section 7.

The next theorem provides sharp upper bounds on shifted Dirichlet eigenvalues of the Pauli operator.

Theorem 3.1 (Dirichlet-Pauli operator). Theorem 2.1 and Corollarly 2.2 hold with the Dirichlet eigenvalues of the magnetic Laplacian replaced by shifted Dirichlet eigenvalues of the Pauli operator; namely, replace $\lambda_{j}$ with $\lambda_{j}^{P}+\beta / A$.

We do not have an analogous result for the Neumann eigenvalues of the Pauli operator. Indeed, the Pauli operator does not have discrete spectrum on $H^{1}$, because its null space is infinite dimensional; see the discussion in Section 7.

\section{ThE CONSTANT JACOBIAN TRANSFORMATION}

In Figure 2 we showed how to construct a mapping from a starlike domain of area $\pi$ to the unit disk: we choose the map to be linear on each ray, with the angular deformation of rays determined by requiring that the mapping should preserve the area of each infinitesimal sector.

For a general starlike domain $\Omega$, we simply rescale the formula from Figure 2 as we did already in our work on the Laplacian [18]. More precisely, we determine the angular deformation $\phi(\theta)$ by integrating the initial value problem

$$
\phi^{\prime}(\theta)=R(\theta)^{2} \frac{\pi}{A}, \quad \phi(0)=0 .
$$


Notice $\phi$ increases by $2 \pi$ as $\theta$ increases by $2 \pi$, since $\int_{0}^{2 \pi} R(\theta)^{2} \mathrm{~d} \theta=2 A$. Then we define a transformation $T: \Omega \rightarrow \mathbb{D}$ from the starlike domain to the unit disk by working in polar coordinates:

$$
T:(r, \theta) \mapsto(r / R(\theta), \phi(\theta)) .
$$

Obviously the transformation is linear with respect to $r$, on each ray, and one easily checks that the Jacobian is constant, with $\operatorname{Jac}(T) \equiv \pi / A$.

\section{Dirichlet eigenvalues - Proof of Theorem 2.1 And Corollary 2.2}

(Throughout the section, the changes needed for the Neumann case in Theorem 2.5 will be indicated in parentheses, like this.)

It suffices to prove the theorem for the identity function $\Phi(a)=a$, because then Hardy-Littlewood-Pólya majorization extends the result to all concave increasing $\Phi$. (For references on majorization, see [18], Appendix A).

Thus we must investigate the partial sum of eigenvalues, that is, $\sum_{j=1}^{n} \lambda_{j}$.

In order to get upper bounds, our task is to choose suitable trial functions in a variational characterization of the eigenvalue sum. We will construct trial functions on $\Omega$ by transplanting eigenfunctions from the disk with the help of the area-preserving map $T$ constructed in the previous section. This method does not require explicit formulas for the eigenfunctions on the disk. Then we average with respect to all pre-rotations of the disk.

The Rayleigh quotient associated with the Dirichlet spectrum (1.2) of the magnetic Laplacian is

$$
\operatorname{Ray}[v]=\frac{\int_{\Omega}\left|\left(i \nabla+\frac{\beta}{2 A}\left(-x_{2}, x_{1}\right)\right) v\right|^{2} \mathrm{~d} x}{\int_{\Omega}|v|^{2} \mathrm{~d} x} \quad \text { for } v \in H_{0}^{1}(\Omega ; \mathbb{C}) .
$$

(To handle the Neumann spectrum in Theorem 2.5 one would simply replace $H_{0}^{1}$ with $H^{1}$.) To express the numerator in polar coordinates we write $\mathbf{e}_{r}$ and $\mathbf{e}_{\theta}$ for the radial and angular unit vectors, and write $v_{r}$ and $v_{\theta}$ for the derivatives of $v$ with respect to $r$ and $\theta$. Then

$$
\begin{aligned}
\operatorname{Ray}[v] & =\frac{\int_{\Omega}\left|i v_{r} \mathbf{e}_{r}+i r^{-1} v_{\theta} \mathbf{e}_{\theta}+\frac{\beta}{2 A} v r \mathbf{e}_{\theta}\right|^{2} \mathrm{~d} x}{\int_{\Omega}|v|^{2} \mathrm{~d} x} \\
& =\frac{\int_{\Omega}\left\{\left|v_{r}\right|^{2}+\left|i r^{-1} v_{\theta}+\frac{\beta}{2 A} r v\right|^{2}\right\} r \mathrm{~d} r \mathrm{~d} \theta}{\int_{\Omega}|v|^{2} r \mathrm{~d} r \mathrm{~d} \theta} .
\end{aligned}
$$

The Rayleigh-Poincaré Variational Principle ([3], p. 98) characterizes the partial sum of the Dirichlet eigenvalues as:

$$
\begin{aligned}
\lambda_{1}+\cdots+\lambda_{n}=\min \left\{\operatorname{Ray}\left[v_{1}\right]+\cdots+\operatorname{Ray}\left[v_{n}\right]:\right. \\
\left.v_{1}, \ldots, v_{n} \in H_{0}^{1}(\Omega ; \mathbb{C}) \text { are pairwise orthogonal in } L^{2}(\Omega ; \mathbb{C})\right\} .
\end{aligned}
$$

(Again, for the Neumann eigenvalues one would replace $H_{0}^{1}$ with $H^{1}$.) To apply this principle, we let $u_{1}, u_{2}, u_{3}, \ldots$ be orthonormal eigenfunctions on the unit disk $\mathbb{D}$ corresponding to the eigenvalues $\lambda_{1}(\mathbb{D}), \lambda_{2}(\mathbb{D}), \lambda_{3}(\mathbb{D}), \ldots$ Let $\eta \in \mathbb{R}$ and use $U$ to denote rotation of the plane by angle $\eta$. Then define trial functions on $\Omega$ by

$$
v_{j}=u_{j} \circ U^{-1} \circ T
$$

where the transformation $T: \Omega \rightarrow \mathbb{D}$ was defined in Section 4. Thus in polar coordinates we have

$$
v_{j}(r, \theta)=u_{j}(r / R(\theta), \phi(\theta)-\eta) .
$$


One can show that $v_{j} \in H^{1} \cap C(\Omega)$, by using that $R(\theta)$ is Lipschitz and $\phi(\theta)$ is continuously differentiable. Further, $v_{j}=0$ on the boundary of $\Omega$ because $u_{j}=0$ on the boundary of the disk, and thus $v_{j} \in H_{0}^{1}(\Omega)$. (This last step is unnecessary for the Neumann case, since no boundary condition need be imposed on the trial functions in $H^{1}$.)

The functions $v_{j}$ are pairwise orthogonal, since

$$
\begin{aligned}
\int_{\Omega} v_{j} \overline{v_{k}} \mathrm{~d} x & =\operatorname{Jac}\left(T^{-1}\right) \int_{\mathbb{D}} u_{j} \overline{u_{k}} \mathrm{~d} x \\
& =0
\end{aligned}
$$

whenever $j \neq k$, using here that $u_{j}$ and $u_{k}$ are orthogonal and $T^{-1}$ has constant Jacobian. Thus by the Rayleigh-Poincaré principle, we have

$$
\sum_{j=1}^{n} \lambda_{j}(\Omega) \leq \sum_{j=1}^{n} \frac{\int_{\Omega}\left|\left(i \nabla+\frac{\beta}{2 A}\left(-x_{2}, x_{1}\right)\right) v_{j}\right|^{2} \mathrm{~d} x}{\int_{\Omega}\left|v_{j}\right|^{2} \mathrm{~d} x} .
$$

The denominator of this Rayleigh quotient is $\int_{\Omega}\left|v_{j}\right|^{2} \mathrm{~d} x=\operatorname{Jac}\left(T^{-1}\right)=A / \pi$ by (5.3) with $j=k$, since the eigenfunctions are normalized with $\int_{\mathbb{D}}\left|u_{j}\right|^{2} \mathrm{~d} x=1$.

To evaluate the numerator of the Rayleigh quotient, we develop some lemmas. Write $u=u_{j}$ and $v=v_{j}$, to simplify notation in what follows, and express $u$ and $v$ in polar coordinates as $u(s, \phi)$ and $v(r, \theta)$, respectively. These functions are related by (5.2).

\section{Lemma 5.1.}

$$
\left|v_{r}(r, \theta)\right|^{2} R(\theta)^{2}=\left|u_{s}(r / R(\theta), \phi(\theta)-\eta)\right|^{2} .
$$

Proof. Simply differentiate (5.2) with respect to $r$, and square the result.

\section{Lemma 5.2.}

$$
\begin{aligned}
& \left|i r^{-1} v_{\theta}+\frac{\beta}{2 A} r v\right|^{2} R(\theta)^{2} \\
= & \left|u_{s}(r / R(\theta), \phi(\theta)-\eta)\right|^{2}(\log R)^{\prime}(\theta)^{2}+2 \operatorname{Re} \overline{u_{s}(r / R(\theta), \phi(\theta)-\eta)} \\
& \times\left(-\frac{R(\theta)}{r} u_{\phi}(r / R(\theta), \phi(\theta)-\eta)+\frac{i \beta}{2 \pi} \frac{r}{R(\theta)} u(r / R(\theta), \phi(\theta)-\eta)\right) \frac{\pi}{A} R(\theta) R^{\prime}(\theta) \\
& +\left|i \frac{R(\theta)}{r} u_{\phi}(r / R(\theta), \phi(\theta)-\eta)+\frac{\beta}{2 \pi} \frac{r}{R(\theta)} u(r / R(\theta), \phi(\theta)-\eta)\right|^{2} \frac{\pi^{2} R(\theta)^{4}}{A^{2}} .
\end{aligned}
$$

Proof. Differentiating (5.2) with respect to $\theta$ gives that

$$
v_{\theta}(r, \theta)=-r u_{s}(r / R(\theta), \phi(\theta)-\eta) R^{\prime}(\theta) / R(\theta)^{2}+u_{\phi}(r / R(\theta), \phi(\theta)-\eta) \phi^{\prime}(\theta) .
$$

Substituting this formula into the left side of the lemma yields an expression of the form $|a+b+c|^{2}$, which we expand as $|a|^{2}+2 \operatorname{Re} \bar{a}(b+c)+|b+c|^{2}$, hence obtaining the right side of the lemma. In the final simplification we use also that $\phi^{\prime}(\theta)=R(\theta)^{2} \pi / A$.

Lemma 5.3. The numerator of the Rayleigh quotient for $v$ is

$$
\int_{\Omega}\left|\left(i \nabla+\frac{\beta}{2 A}\left(-x_{2}, x_{1}\right)\right) v\right|^{2} \mathrm{~d} x=Q_{1}+Q_{2}+Q_{3}
$$


where

$$
\begin{aligned}
Q_{1}= & \int_{0}^{2 \pi} \int_{0}^{1}\left|u_{s}(s, \phi(\theta)-\eta)\right|^{2} s \mathrm{~d} s\left[1+(\log R)^{\prime}(\theta)^{2}\right] \mathrm{d} \theta, \\
Q_{2}= & 2 \operatorname{Re} \int_{0}^{2 \pi} \int_{0}^{1} \overline{u_{s}(s, \phi(\theta)-\eta)} \\
& \times\left(-s^{-1} u_{\phi}(s, \phi(\theta)-\eta)+\frac{i \beta}{2 \pi} s u(s, \phi(\theta)-\eta)\right) s \mathrm{~d} s \frac{\pi}{A} R(\theta) R^{\prime}(\theta) \mathrm{d} \theta, \\
Q_{3}= & \int_{0}^{2 \pi} \int_{0}^{1}\left|i s^{-1} u_{\phi}(s, \phi(\theta)-\eta)+\frac{\beta}{2 \pi} s u(s, \phi(\theta)-\eta)\right|^{2} s \mathrm{~d} s \frac{\pi^{2} R(\theta)^{4}}{A^{2}} \mathrm{~d} \theta .
\end{aligned}
$$

Proof. Start with the numerator in polar coordinates as in (5.1), then substitute using Lemmas 5.1 and 5.2, and make the radial change of variable $r=s R(\theta)$, so that $0<s<1$.

Lemma 5.4. The averages of $Q_{1}, Q_{2}, Q_{3}$ with respect to $\eta$ are:

$$
\begin{aligned}
& \frac{1}{2 \pi} \int_{0}^{2 \pi} Q_{1} \mathrm{~d} \eta=G_{0}(\Omega) \int_{\mathbb{D}}\left|u_{s}\right|^{2} \mathrm{~d} x, \\
& \frac{1}{2 \pi} \int_{0}^{2 \pi} Q_{2} \mathrm{~d} \eta=0, \\
& \frac{1}{2 \pi} \int_{0}^{2 \pi} Q_{3} \mathrm{~d} \eta=G_{1}(\Omega) \int_{\mathbb{D}}\left|i s^{-1} u_{\phi}+\frac{\beta}{2 \pi} s u\right|^{2} \mathrm{~d} x .
\end{aligned}
$$

Proof. For $Q_{1}$, we integrate the definition in Lemma 5.3 with respect to $\eta$ and interchange the order of integration. Then making the substitution $\eta \mapsto \phi(\theta)-\eta$ allows us to separate the $\eta$ and $\theta$ integrals, which completes the proof when we recall the definition of $G_{0}$ from the Introduction. The argument is analogous for $Q_{3}$.

With $Q_{2}$ we proceed similarly, and then observe that $\int_{0}^{2 \pi} R(\theta) R^{\prime}(\theta) \mathrm{d} \theta=\left.\frac{1}{2} R(\theta)^{2}\right|_{0} ^{2 \pi}=0$ by periodicity.

Remark 5.5. Our proof that the cross-term $Q_{2}$ vanishes after averaging with respect to $\eta$ seems like a trick since it relies on the quantity $R R^{\prime}$ being a derivative of a periodic function. To avoid using this fact, one may include a reflection as well as rotations when constructing trial functions, as follows. Write $\Pi$ for reflection in the horizontal axis, and consider the additional trial functions

$$
w_{j}=\overline{u_{j}} \circ \Pi \circ U^{-1} \circ T,
$$

which in polar coordinates can be written

$$
w_{j}(r, \theta)=\overline{u_{j}(r / R(\theta), \eta-\phi(\theta))} .
$$

Now carry out the proof as above, except using $w_{j}$ instead of $v_{j}$. The resulting quantities $Q_{1}$ and $Q_{3}$ are the same as for $v_{j}$, but $Q_{2}$ acquires a negative sign in front. Hence by averaging the numerators of the Rayleigh quotients for $v_{j}$ and $w_{j}$ we eliminate $Q_{2}$ and obtain simply $Q_{1}+Q_{3}$. Then one averages with respect to $\eta$ by the formulas in Lemma 5.4 .

Now we return to the proof of the theorem. The left side of (5.4) is independent of the rotation angle $\eta$. Hence by averaging (5.4) with respect to $\eta \in[0,2 \pi]$ we find

$$
\sum_{j=1}^{n} \lambda_{j}(\Omega) \leq \sum_{j=1}^{n} \frac{\frac{1}{2 \pi} \int_{0}^{2 \pi}\left(Q_{1}+Q_{2}+Q_{3}\right) \mathrm{d} \eta}{A / \pi},
$$


where we must remember that " $u$ " means $u_{j}$, in the quantities $Q_{1}, Q_{2}, Q_{3}$. Thus Lemma 5.4 shows that

$$
\begin{aligned}
\sum_{j=1}^{n} \lambda_{j}(\Omega) A & \leq \pi \sum_{j=1}^{n}\left[G_{0}(\Omega) \int_{\mathbb{D}}\left|\frac{\partial u_{j}}{\partial s}\right|^{2} \mathrm{~d} x+G_{1}(\Omega) \int_{\mathbb{D}}\left|i s^{-1} \frac{\partial u_{j}}{\partial \phi}+\frac{\beta}{2 \pi} s u_{j}\right|^{2} \mathrm{~d} x\right] \\
& =\pi \sum_{j=1}^{n}\left[\left(1-\alpha_{j}\right) G_{0}(\Omega)+\alpha_{j} G_{1}(\Omega)\right] \int_{\mathbb{D}}\left|\left(i \nabla+\frac{\beta}{2 \pi}\left(-x_{2}, x_{1}\right)\right) u_{j}\right|^{2} \mathrm{~d} x
\end{aligned}
$$

where

$$
\alpha_{j}=\frac{\int_{\mathbb{D}}\left|i s^{-1} \frac{\partial u_{j}}{\partial \phi}+\frac{\beta}{2 \pi} s u_{j}\right|^{2} \mathrm{~d} x}{\int_{\mathbb{D}}\left|\left(i \nabla+\frac{\beta}{2 \pi}\left(-x_{2}, x_{1}\right)\right) u_{j}\right|^{2} \mathrm{~d} x}, \quad j=1,2, \ldots, n .
$$

The coefficient $\alpha_{j} \in[0,1]$ measures the "angular component" of the magnetic energy of the $j$ th mode; see (5.1).

We may estimate $G_{0}$ and $G_{1}$ from above with their maximum, $G$, so that

$$
\sum_{j=1}^{n} \lambda_{j}(\Omega) A(\Omega) / G(\Omega) \leq \pi \sum_{j=1}^{n} \int_{\mathbb{D}}\left|\left(i \nabla+\frac{\beta}{2 \pi}\left(-x_{2}, x_{1}\right)\right) u_{j}\right|^{2} \mathrm{~d} x=\pi \sum_{j=1}^{n} \lambda_{j}(\mathbb{D}) .
$$

Since $A(\mathbb{D})=\pi$ and $G(\mathbb{D})=1$, Theorem 2.1 is proved for the case when $\Phi(a)=a$ is the identity function, as desired. (We have proved the theorem with a unit disk on the right side, but then it holds for any centered disk by scale invariance.)

\subsection{Proof of Corollary 2.2}

The function $\Phi(a)=a^{s}$ is concave and increasing, when $0<s \leq 1$, and this choice of $\Phi$ in Theorem 2.1 gives maximality of $\left(\lambda_{1}^{s}+\ldots+\lambda_{n}^{s}\right)^{1 / s} A / G$ for the centered disk. Choosing $\Phi(a)=\log a$ shows maximality of the centered disk for the functional

$$
\sum_{j=1}^{n} \log \left(\lambda_{j} A / G\right)=n \log \left(\sqrt[n]{\lambda_{1} \ldots, \lambda_{n}} A / G\right) .
$$

The function $\Phi(a)=-a^{s}$ is concave increasing, when $s<0$, and so we obtain minimality of the centered disk for $\sum_{j=1}^{n}\left(\lambda_{j} A / G\right)^{s}$. Lastly, for $t>0$ we consider $\Phi(a)=-\mathrm{e}^{-a t}$ to prove minimality of $\sum_{j=1}^{n} \exp \left(-\lambda_{j} A t / G\right)$ for the centered disk.

\section{Dirichlet equality statement.}

Assume equality holds for the first eigenvalue, that is,

$$
\lambda_{1} A /\left.G\right|_{\Omega}=\lambda_{1} A /\left.G\right|_{\mathbb{D}} .
$$

By enforcing equality in the proof of Theorem 2.1, with $n=1$, we see that the trial function $v_{1}$ on $\Omega$ must attain equality in the Rayleigh characterization of $\lambda_{1}(\Omega)$, and hence must be a first eigenfunction for $\Omega$. In particular this holds when $\eta=0$ (no rotation), so that the function $v(x)=u_{1}(T(x))$ satisfies

$$
(i \nabla+F)^{2} v=\lambda_{1}(\Omega) v
$$

classically, where $F(x)=\frac{\beta}{2 A}\left(-x_{2}, x_{1}\right)$. That is,

$$
-\Delta v+2 i F \cdot \nabla v+|F|^{2} v=\lambda_{1}(\Omega) v .
$$


The ground state of the disk is real-valued and radial, for the magnetic Laplacian with Dirichlet boundary condition, by work of Erdös ([6], Appendix), or see the more explicit treatment by Son ([23], Thm. 3.3.4). Hence $u_{1}(x)=J(|x|)$ for some real-valued function $J$. (The precise form of $J$ is unimportant here. As it happens, $J$ for the Laplacian is simply the zeroth Bessel function, whereas for the magnetic Laplacian it turns out to be a confluent hypergeometric or "Kummer" function.) Observe that $J^{\prime}\left(r_{0}\right) \neq 0$ for some $r_{0} \in(0,1)$ because $J$ cannot be identically zero and $J(1)=0$ by the Dirichlet boundary condition.

Taking imaginary parts in (5.6) shows that $F \cdot \nabla v=0$, so that $\beta v_{\theta}=0$. Suppose $\beta>0$. Since $v(x)=$ $u_{1}(T(x))=J(r / R(\theta))$, we have

$$
0=v_{\theta}=-J^{\prime}(r / R(\theta)) r R(\theta)^{-2} R^{\prime}(\theta) .
$$

Choosing $r=r_{0} R(\theta)$, we deduce that $R^{\prime}(\theta)=0$ for almost every $\theta$. Hence the radius function is constant, which means $\Omega$ is a centered disk.

For the equality case when $\beta=0$, see our earlier work ([18], Thm. 3.1). That earlier work assumes $R(\theta)$ is $C^{2}$-smooth, but in fact that smoothness follows from inverting the formula $v(x)=J(r / R(\theta))$ to solve for $R$, using smoothness of the first eigenfunction $v$ and the radial function $J$.

\section{Perturbation analysis}

\subsection{Proof of Corollary 2.3}

Start by applying Theorem 2.1 to the first eigenvalue $(n=1)$ and then substitute $R=1+\varepsilon P$ into the definitions of $G_{0}$ and $G_{1}$. One obtains the following expressions:

$$
\begin{aligned}
G_{0}\left(\Omega_{\varepsilon}\right)=1+\int_{0}^{2 \pi} \frac{\varepsilon^{2} P^{\prime}(\theta)^{2}}{(1+\varepsilon P(\theta))^{2}} \frac{\mathrm{d} \theta}{2 \pi} & =1+\varepsilon^{2} \int_{0}^{2 \pi} P^{\prime}(\theta)^{2} \frac{\mathrm{d} \theta}{2 \pi}+O\left(\varepsilon^{3}\right) \\
& =1+\varepsilon^{2} \sum_{n \neq 0} n^{2}\left|p_{n}\right|^{2}+O\left(\varepsilon^{3}\right)
\end{aligned}
$$

and

$$
\begin{aligned}
G_{1}\left(\Omega_{\varepsilon}\right)=\frac{\int_{0}^{2 \pi}(1+\varepsilon P(\theta))^{4} \mathrm{~d} \theta / 2 \pi}{\left[\int_{0}^{2 \pi}(1+\varepsilon P(\theta))^{2} \mathrm{~d} \theta / 2 \pi\right]^{2}} & =1+4 \varepsilon^{2} \int_{0}^{2 \pi}\left(P(\theta)-p_{0}\right)^{2} \frac{\mathrm{d} \theta}{2 \pi}+O\left(\varepsilon^{3}\right) \\
& =1+4 \varepsilon^{2} \sum_{n \neq 0}\left|p_{n}\right|^{2}+O\left(\varepsilon^{3}\right) .
\end{aligned}
$$

The upper bound in the corollary now follows once we use the symmetry of the coefficients $\left(p_{-n}=\overline{p_{n}}\right)$.

\subsection{Proof of formal asymptotic (2.1)}

The Kummer function $M(a, b, z)$ satisfies the differential equation

$$
z M^{\prime \prime}+(b-z) M^{\prime}-a M=0
$$

with initial condition $M(a, b, 0)=1$. (Recall that primes indicate derivatives with respect to $z$.) Define

$$
f_{n}(r, \lambda)=\left(r^{2} / \pi\right)^{|n| / 2} e^{-\beta r^{2} / 4 \pi} M\left((1+|n|-n-\lambda / \beta) / 2,|n|+1, \frac{\beta r^{2}}{2 \pi}\right)
$$

for $n \in \mathbb{Z}$ and $r, \lambda>0$. We rescale $f_{n}$ by area, then modulate by $\mathrm{e}^{\mathrm{i} n \theta}$, and form a series combination as follows:

$$
u(r, \theta)=f_{0}(r \sqrt{\pi / A}, \lambda A)+\varepsilon \sum_{n \neq 0} c_{n} f_{n}(r \sqrt{\pi / A}, \lambda A) \mathrm{e}^{\mathrm{i} n \theta},
$$


where the coefficients $c_{n}$ are undetermined for now. Assuming that the series converges suitably, one computes that $u$ formally satisfies the eigenvalue equation $(i \nabla+F)^{2} u=\lambda u$ in the plane, by expressing the eigenvalue equation in polar coordinates as

$$
-\left(u_{r r}+r^{-1} u_{r}+r^{-2} u_{\theta \theta}\right)+i \frac{\beta}{A} u_{\theta}+\frac{\beta^{2} r^{2}}{4 A^{2}} u=\lambda u
$$

and then substituting the series for $u$ and using the Kummer differential equation.

The goal of the perturbation analysis is to choose the parameter $\lambda$ and coefficients $c_{n}$ so that $u=0$ on the boundary of the perturbed domain $\Omega_{\varepsilon}$, to second order in $\varepsilon$. (The resulting analysis is only "formal", since we neglect terms of third order and higher.)

Recall $\Omega_{\varepsilon}$ is a nearly circular domain defined in polar coordinates by $r \leq R(\theta)=1+\varepsilon P(\theta)$, where $P$ is expressed in a Fourier series as before. We assume the constant term vanishes: $p_{0}=0$. Let

$$
\gamma=\sum_{n \neq 0}\left|p_{n}\right|^{2}=\frac{1}{2 \pi} \int_{0}^{2 \pi} P(\theta)^{2} \mathrm{~d} \theta
$$

so that the domain has area

$$
A=\frac{1}{2} \int_{0}^{2 \pi} R(\theta)^{2} \mathrm{~d} \theta=\pi\left(1+\varepsilon^{2} \gamma\right)
$$

and hence

$$
\begin{gathered}
\sqrt{\frac{\pi}{A}}=1-\varepsilon^{2} \gamma / 2+O\left(\varepsilon^{4}\right), \\
R(\theta) \sqrt{\frac{\pi}{A}}=1+\varepsilon P(\theta)-\varepsilon^{2} \gamma / 2+O\left(\varepsilon^{3}\right) .
\end{gathered}
$$

Denote by $\lambda_{0}$ the lowest magnetic eigenvalue of the unit disk, with Dirichlet boundary condition. The corresponding ground state on the disk is the radial function $f_{0}\left(r, \lambda_{0} \pi\right)$, satisfying

$$
-\left(u_{r r}+r^{-1} u_{r}\right)+\frac{\beta^{2} r^{2}}{4 \pi^{2}} u=\lambda_{0} u
$$

with the boundary condition

$$
f_{0}\left(1, \lambda_{0} \pi\right)=0, \quad \text { or } \quad M\left(a_{0}, 1, z\right)=0
$$

where $z=\beta / 2 \pi$. This claim that the ground state arises from the radial case $n=0$ can be found in work of Erdös ([6], Appendix), or later Son ([23], Thm. 3.3.4). The formula $f_{0}\left(r, \lambda_{0} \pi\right)$ for the radial ground state comes from ([23], Eq. (3.5.9)) with $n=0$, except with $\beta$ there replaced by $\beta / 2 \pi$ to match the vector potential in this paper, and using that $E$ in that paper equals the eigenvalue $\lambda$ when $n=0$ (see [23], Sect. 4.2).

To carry out a perturbation analysis, we assume that the lowest eigenvalue of the perturbed domain varies with $\varepsilon$ according to

$$
\lambda A=\lambda_{0} \pi+\rho \varepsilon+\tau \varepsilon^{2}+O\left(\varepsilon^{3}\right),
$$

for some coefficients $\rho$ and $\tau$ to be determined. For the Dirichlet boundary condition on $\Omega_{\varepsilon}$ we require

$$
0=u(R(\theta), \theta), \quad \theta \in[0,2 \pi],
$$

so that we want

$$
\begin{aligned}
0= & f_{0}\left(1+\varepsilon P(\theta)-\varepsilon^{2} \gamma / 2+O\left(\varepsilon^{3}\right), \lambda_{0} \pi+\rho \varepsilon+\tau \varepsilon^{2}+O\left(\varepsilon^{3}\right)\right) \\
& +\varepsilon \sum_{n \neq 0} c_{n} f_{n}\left(1+\varepsilon P(\theta)-\varepsilon^{2} \gamma / 2+O\left(\varepsilon^{3}\right), \lambda_{0} \pi+\rho \varepsilon+\tau \varepsilon^{2}+O\left(\varepsilon^{3}\right)\right) \mathrm{e}^{\mathrm{i} n \theta} .
\end{aligned}
$$


Denote the partial derivatives of $f_{n}$ using superscripts, so that $f_{n}^{1,0}=\partial f_{n} / \partial r$. Then the Taylor expansion of the above boundary condition says to second order in $\varepsilon$ that

$$
\begin{aligned}
0= & f_{0}\left(1, \lambda_{0} \pi\right)+\varepsilon \sum_{n \neq 0} c_{n} f_{n}\left(1, \lambda_{0} \pi\right) \mathrm{e}^{\mathrm{i} n \theta} \\
& +\left(\varepsilon P(\theta)-\frac{\varepsilon^{2}}{2} \gamma\right) f_{0}^{1,0}\left(1, \lambda_{0} \pi\right)+\left(\varepsilon \rho+\tau \varepsilon^{2}\right) f_{0}^{0,1}\left(1, \lambda_{0} \pi\right) \\
& +\varepsilon \sum_{n \neq 0} c_{n}\left(\varepsilon P(\theta) f_{n}^{1,0}\left(1, \lambda_{0} \pi\right)+\varepsilon \rho f_{n}^{0,1}\left(1, \lambda_{0} \pi\right)\right) \mathrm{e}^{\mathrm{i} n \theta} \\
& +\frac{1}{2} \varepsilon^{2} P^{2}(\theta) f_{0}^{2,0}\left(1, \lambda_{0} \pi\right)+\varepsilon^{2} \rho P(\theta) f_{0}^{1,1}\left(1, \lambda_{0} \pi\right)+\frac{1}{2} \varepsilon^{2} \rho^{2} f_{0}^{0,2}\left(1, \lambda_{0} \pi\right)+O\left(\varepsilon^{3}\right) .
\end{aligned}
$$

The zeroth order term vanishes by (6.3). We want the first and the second order terms to vanish also. Vanishing of the first order term requires

$$
\sum_{n \neq 0} c_{n} f_{n}\left(1, \lambda_{0} \pi\right) \mathrm{e}^{\mathrm{i} n \theta}+\sum_{n \neq 0} p_{n} \mathrm{e}^{\mathrm{i} n \theta} f_{0}^{1,0}\left(1, \lambda_{0} \pi\right)+\rho f_{0}^{0,1}\left(1, \lambda_{0} \pi\right)=0 .
$$

The constant term in this equation tells us that $\rho f_{0}^{0,1}\left(1, \lambda_{0} \pi\right)=0$, and hence

$$
\rho=0
$$

(assuming for now that $f_{0}^{0,1}\left(1, \lambda_{0} \pi\right) \neq 0$, which we justify below). For $n \neq 0$, we get

$$
c_{n}=-p_{n} \frac{f_{0}^{1,0}\left(1, \lambda_{0} \pi\right)}{f_{n}\left(1, \lambda_{0} \pi\right)} .
$$

If we average the second order term over $\theta$ and put $\rho=0$, we get

$$
\tau f_{0}^{0,1}\left(1, \lambda_{0} \pi\right)+\sum_{n \neq 0} c_{n} p_{-n} f_{n}^{1,0}\left(1, \lambda_{0} \pi\right)+\frac{\gamma}{2} f_{0}^{2,0}\left(1, \lambda_{0} \pi\right)-\frac{\gamma}{2} f_{0}^{1,0}\left(1, \lambda_{0} \pi\right)=0 .
$$

Note that $f_{0}^{2,0}\left(1, \lambda_{0} \pi\right)=-f_{0}^{1,0}\left(1, \lambda_{0} \pi\right)$ by evaluating the eigenvalue equation (6.2) at $r=1$. Hence using (6.4), we may solve for the coefficient $\tau$ as

$$
\begin{aligned}
\tau & =\frac{f_{0}^{1,0}\left(1, \lambda_{0} \pi\right)}{f_{0}^{0,1}\left(1, \lambda_{0} \pi\right)} \sum_{n \neq 0}\left|p_{n}\right|^{2}\left(1+\frac{f_{n}^{1,0}\left(1, \lambda_{0} \pi\right)}{f_{n}\left(1, \lambda_{0} \pi\right)}\right) \\
& =2 \frac{f_{0}^{1,0}\left(1, \lambda_{0} \pi\right)}{f_{0}^{0,1}\left(1, \lambda_{0} \pi\right)} \sum_{n=1}^{\infty}\left|p_{n}\right|^{2}\left(1+\frac{1}{2} \frac{f_{n}^{1,0}\left(1, \lambda_{0} \pi\right)}{f_{n}\left(1, \lambda_{0} \pi\right)}+\frac{1}{2} \frac{f_{-n}^{1,0}\left(1, \lambda_{0} \pi\right)}{f_{-n}\left(1, \lambda_{0} \pi\right)}\right)
\end{aligned}
$$

by symmetry, since $\left|p_{n}\right|=\left|p_{-n}\right|$.

Let us simplify these expressions. The ratio before the infinite sum in (6.5) evaluates to

$$
2 \frac{f_{0}^{1,0}\left(1, \lambda_{0} \pi\right)}{f_{0}^{0,1}\left(1, \lambda_{0} \pi\right)}=2 \frac{M^{\prime}\left(a_{0}, 1, z\right) \cdot \beta / \pi}{\frac{\partial M}{\partial a}\left(a_{0}, 1, z\right) \cdot(-1 / 2 \beta)}=c,
$$

by definition of $f_{0}$ and $c$ and remembering that $M\left(a_{0}, 1, z\right)=0$ from (6.3).

Next, write $q_{n}$ for the factor (..) in (6.5). By substituting the definitions of $f_{n}$ and $f_{-n}$ into (6.5) we see that

$$
q_{n}=1+n-z+z(\log M)^{\prime}\left(a_{0}, n+1, z\right)+z(\log M)^{\prime}\left(a_{0}+n, n+1, z\right),
$$


where the derivatives are evaluated at the specific value $z=\beta / 2 \pi$ and we recall the definition $a_{0}=\left(1-\lambda_{0} \pi / \beta\right) / 2$. Thus $q_{n}$ has the form claimed in the theorem.

We will show the first coefficient vanishes: $q_{1}=0$. Start with the definition

$$
q_{1}=2-z+z(\log M)^{\prime}\left(a_{0}, 2, z\right)+z(\log M)^{\prime}\left(a_{0}+1,2, z\right) .
$$

First observe that $a_{0}<0$, since $\lambda_{0}>\beta / \pi$ (either by domain monotonicity and comparison with the first Landau level on the plane, or else by [23], Thm. 3.3.4). Then note that

$$
M\left(a_{0}+1,2, \cdot\right)=\frac{1}{a_{0}} M^{\prime}\left(a_{0}, 1, \cdot\right)
$$

by the identity ([20], Eq. (13.3.15)). Further,

$$
M\left(a_{0}, 2, \cdot\right)=\frac{1}{a_{0}-1}\left(M^{\prime}\left(a_{0}, 1, \cdot\right)-M\left(a_{0}, 1, \cdot\right)\right)
$$

by expanding ([20], Eq. (13.3.20)). Substituting these last two formulas into the expression above for $q_{1}$ shows that

$$
q_{1}=2-z+z \frac{M^{\prime \prime}\left(a_{0}, 1, z\right)-M^{\prime}\left(a_{0}, 1, z\right)}{M^{\prime}\left(a_{0}, 1, z\right)-M\left(a_{0}, 1, z\right)}+z \frac{M^{\prime \prime}\left(a_{0}, 1, z\right)}{M^{\prime}\left(a_{0}, 1, z\right)} .
$$

Since $M\left(a_{0}, 1, z\right)=0$ by $(6.3)$, we may simplify to obtain

$$
q_{1}=2-2 z+2 \frac{z M^{\prime \prime}\left(a_{0}, 1, z\right)}{M^{\prime}\left(a_{0}, 1, z\right)} .
$$

The Kummer differential equation (6.1) allows us to substitute for $M^{\prime \prime}$, leading to

$$
q_{1}=2-2 z+2 \frac{(z-1) M^{\prime}\left(a_{0}, 1, z\right)}{M^{\prime}\left(a_{0}, 1, z\right)}=0
$$

as we wanted to show.

Finally we show $q_{n}=n+O(1)$. First, for any fixed $a, b, \zeta \in \mathbb{R}$ we have

$$
M(a, n+b, \zeta) \rightarrow 1 \quad \text { and } \quad M^{\prime}(a, n+b, \zeta) \rightarrow 0
$$

as $n \rightarrow \infty$, by the series definition of the Kummer function [20], [13.2.2]. Second,

$$
M(n+a, n+b, \zeta) \rightarrow e^{\zeta} \quad \text { and } \quad M^{\prime}(n+a, n+b, \zeta) \rightarrow e^{\zeta}
$$

as $n \rightarrow \infty$, again by using the series for the Kummer function. Hence the definition (6.6) implies that $q_{n}=$ $n+1+o(1)$ as $n \rightarrow \infty$.

\section{Pauli eigenvalues}

\subsection{Ellipticity of the numerator}

The numerator of the Pauli-Rayleigh quotient (3.1) decouples as follows.

Lemma 7.1 (Decoupling of numerator). For $\psi=\left(\begin{array}{c}\psi_{+} \\ \psi_{-}\end{array}\right)$belonging to $H_{0}^{1}\left(\Omega ; \mathbb{C}^{2}\right)$ we have

$$
\int_{\Omega}\left|\sum_{m=1}^{2} \sigma_{m}\left(i \partial_{m}+F_{m}\right) \psi\right|^{2} \mathrm{~d} x=\int_{\Omega}\left(\left|(i \nabla+F) \psi_{+}\right|^{2}-\frac{\beta}{A}\left|\psi_{+}\right|^{2}\right) \mathrm{d} x+\int_{\Omega}\left(\left|(i \nabla+F) \psi_{-}\right|^{2}+\frac{\beta}{A}\left|\psi_{-}\right|^{2}\right) \mathrm{d} x .
$$


Proof. We have by direct calculation (using the definition of the Pauli matrices) that

$$
\left|\sum_{m=1}^{2} \sigma_{m}\left(i \partial_{m}+F_{m}\right) \psi\right|^{2}=\left|\left(i \partial_{1}+F_{1}\right) \psi_{+}+i\left(i \partial_{2}+F_{2}\right) \psi_{+}\right|^{2}+\left|\left(i \partial_{1}+F_{1}\right) \psi_{-}-i\left(i \partial_{2}+F_{2}\right) \psi_{-}\right|^{2} .
$$

Expand the squares to obtain

$$
\begin{aligned}
\left|\sum_{m=1}^{2} \sigma_{m}\left(i \partial_{m}+F_{m}\right) \psi\right|^{2}= & \left|(i \nabla+F) \psi_{+}\right|^{2}+2 \operatorname{Re}\left(i \partial_{1}+F_{1}\right) \psi_{+} \overline{i\left(i \partial_{2}+F_{2}\right) \psi_{+}} \\
& +\left|(i \nabla+F) \psi_{-}\right|^{2}-2 \operatorname{Re}\left(i \partial_{1}+F_{1}\right) \psi_{-} \overline{i\left(i \partial_{2}+F_{2}\right) \psi_{-}}
\end{aligned}
$$

Then integrate the first cross-term as follows. One has

$2 \operatorname{Re} \int_{\Omega}\left(i \partial_{1}+F_{1}\right) \psi_{+} \overline{i\left(i \partial_{2}+F_{2}\right) \psi_{+}} \mathrm{d} x=2 \operatorname{Re} \int_{\Omega}\left(-i \partial_{1} \psi_{+} \overline{\partial_{2} \psi_{+}}+\partial_{1} \psi_{+} F_{2} \overline{\psi_{+}}-F_{1} \psi_{+} \overline{\partial_{2} \psi_{+}}-i F_{1} F_{2}\left|\psi_{+}\right|^{2}\right) \mathrm{d} x$

The fourth term in (7.2) is purely imaginary, and so can be discarded. For the first term, we note $\partial_{1} \psi_{+} \overline{\partial_{2} \psi_{+}}$ has real integral because

$$
\overline{\int_{\Omega} \partial_{1} \psi_{+} \overline{\partial_{2} \psi_{+}} \mathrm{d} x}=\int_{\Omega} \partial_{2} \psi_{+} \overline{\partial_{1} \psi_{+}} \mathrm{d} x=\int_{\Omega} \partial_{1} \psi_{+} \overline{\partial_{2} \psi_{+}} \mathrm{d} x
$$

by integration by parts (using the Dirichlet boundary condition). Thus the first term in (7.2) can be discarded too. We are left with the integral of the second and third terms, so that

$$
\begin{aligned}
2 \operatorname{Re} \int_{\Omega}\left(i \partial_{1}+F_{1}\right) \psi_{+} \overline{i\left(i \partial_{2}+F_{2}\right) \psi_{+}} \mathrm{d} x & =\int_{\Omega}\left(F_{2} \partial_{1}\left|\psi_{+}\right|^{2}-F_{1} \partial_{2}\left|\psi_{+}\right|^{2}\right) \mathrm{d} x \\
& =\int_{\Omega}\left(-\partial_{1} F_{2}+\partial_{2} F_{1}\right)\left|\psi_{+}\right|^{2} \mathrm{~d} x \\
& =-\frac{\beta}{A} \int_{\Omega}\left|\psi_{+}\right|^{2} \mathrm{~d} x,
\end{aligned}
$$

where once again the boundary terms have vanished in the integration by parts thanks to the Dirichlet boundary condition. The analogous formula holds for $\psi_{-}$, and so the lemma follows.

Lemma 7.2 (Ellipticity of the numerator). For $\psi=\left(\begin{array}{c}\psi_{+} \\ \psi_{-}\end{array}\right)$belonging to $H_{0}^{1}\left(\Omega ; \mathbb{C}^{2}\right)$,

$$
\int_{\Omega}\left|\sum_{m=1}^{2} \sigma_{m}\left(i \partial_{m}+F_{m}\right) \psi\right|^{2} \mathrm{~d} x \geq \frac{1}{2} \int_{\Omega}|\nabla \psi|^{2} \mathrm{~d} x-C \int_{\Omega}|\psi|^{2} \mathrm{~d} x
$$

where $\nabla \psi=\left(\begin{array}{c}\nabla \psi_{+} \\ \nabla \psi_{-}\end{array}\right)$and the constant can be chosen as $C=\|F\|_{L^{\infty}(\Omega)}^{2}+\beta / A$.

Proof. Combine Lemma 7.1 with the elementary inequality $|a+b|^{2} \geq \frac{1}{2}|a|^{2}-|b|^{2}$. 


\subsection{Proof of Theorem 3.1}

This theorem treats the spectrum of the Pauli operator shifted up by $\beta / A$, that is, it treats the spectrum of $H_{P}+\beta / A$. The Rayleigh quotient for this shifted operator is

$$
\operatorname{Ray}_{\text {shift }}[\psi]=\frac{\int_{\Omega}\left|(i \nabla+F) \psi_{+}\right|^{2} \mathrm{~d} x+\int_{\Omega}\left(\left|(i \nabla+F) \psi_{-}\right|^{2}+\frac{2 \beta}{A}\left|\psi_{-}\right|^{2}\right) \mathrm{d} x}{\int_{\Omega}|\psi|^{2} \mathrm{~d} x},
$$

as we see by adding $\beta / A$ to the definition (3.1) of the Rayleigh quotient for $H_{P}$ and then substituting the expression for its numerator from Lemma 7.1.

Now one may prove the theorem by adapting straightforwardly the proof of Theorem 2.1, using in the course of the proof that $\beta / G \leq \beta$. The equality statement for the first eigenvalue follows immediately from the equality statement in Corollary 2.2, since the lowest Pauli eigenvalue is connected to the lowest eigenvalue of the magnetic Laplacian by $\lambda_{1}^{P}+\beta / A=\lambda_{1}$ (see (7.6) below).

\subsection{Complex form of the Rayleigh quotient}

By substituting $F_{1}=-x_{2} \beta / 2 A$ and $F_{2}=x_{1} \beta / 2 A$ into (7.1) we obtain the complex form of the Rayleigh quotient, which remains valid no matter what boundary conditions $\psi$ might satisfy:

$$
\operatorname{Ray}_{P}[\psi]=4 \frac{\int_{\Omega}\left|(\bar{\partial}+\beta z / 4 A) \psi_{+}\right|^{2}+\left|(\partial-\beta \bar{z} / 4 A) \psi_{-}\right|^{2} \mathrm{~d} x}{\int_{\Omega}|\psi|^{2} \mathrm{~d} x}
$$

where $\partial=\partial / \partial z$ is the complex derivative. Hence the Rayleigh quotient vanishes if and only if

$$
\psi_{+}=e^{-\beta|z|^{2} / 4 A} f_{+}(z) \quad \text { and } \quad \psi_{-}=e^{\beta|z|^{2} / 4 A} \overline{f_{-}(z)}
$$

for some holomorphic functions $f_{+}$and $f_{-}$. We deduce that the zero modes form an infinite dimensional subspace of $H^{1}$, and so the numerator of the Rayleigh quotient is definitely not elliptic on $H^{1}$. To learn about zero modes on the whole plane, readers can consult the Aharanov-Casher theorem [1].

\subsection{Positivity of the first Pauli-Dirichlet eigenvalue}

The first Dirichlet eigenvalue is nonnegative, since the Rayleigh quotient is nonnegative. If the first eigenvalue were zero then the Rayleigh quotient of the first eigenfunction $\psi$ would equal zero, implying (7.3). The holomorphic functions $f_{+}$and $f_{-}$would then be forced to vanish identically, by the Dirichlet boundary condition, and so $\psi \equiv 0$, which is impossible. Hence the first eigenvalue must be positive.

\subsection{Splitting of the spectrum, and an alternative Proof of Theorem 3.1}

The decoupling of the Rayleigh quotient in Lemma 7.1 implies a decoupling of the eigenvalue equations into separate equations for each component of the spinor:

$$
\begin{aligned}
& (i \nabla+F)^{2} \psi_{+}-(\beta / A) \psi_{+}=\lambda \psi_{+} \\
& (i \nabla+F)^{2} \psi_{-}+(\beta / A) \psi_{-}=\lambda \psi_{-} .
\end{aligned}
$$

Hence the introduction of spin into the quantum system splits the spectrum of the Dirichlet magnetic Laplacian into two copies, with one copy shifted up by $\beta / A$ and another shifted down by the same amount. More precisely, if we write $H_{\mathrm{mag}}=(i \nabla+F)^{2}$ for the magnetic Laplacian two dimensions, then the Dirichlet spectrum of the Pauli operator is

$$
\operatorname{spec}\left(H_{P}\right)=\left[\operatorname{spec}\left(H_{\mathrm{mag}}\right)-\frac{\beta}{A}\right] \cup\left[\operatorname{spec}\left(H_{\mathrm{mag}}\right)+\frac{\beta}{A}\right],
$$

with multiplicities being respected by the union. 
One obtains this same result at the level of operators, of course: first expand the definition of the Pauli operator to show that $H_{P}=(i \nabla+F)^{2} I-\sigma \cdot B$, and then use that the magnetic field is vertical to find $\sigma \cdot B=(\beta / A) \sigma_{3}$, which gives (7.4) $-(7.5)$.

We could have proved Theorem 3.1 by using this splitting of the spectrum, as we now explain. Shifting the spectrum up by $\beta / A$ and multiplying by $A / G$ to obtain a scale invariant expression gives

$$
\left(\operatorname{spec}\left(H_{P}\right)+\frac{\beta}{A}\right) \frac{A}{G}=\left[\operatorname{spec}\left(H_{\mathrm{mag}}\right) \frac{A}{G}\right] \cup\left[\operatorname{spec}\left(H_{\mathrm{mag}}\right) \frac{A}{G}+\frac{2 \beta}{G}\right] .
$$

Note that the first spectrum on the right is not shifted, and the second is shifted by an amount $2 \beta / G$ that is maximal for the disk (since $G$ is minimal for the disk). Hence one can prove Theorem 3.1 by starting with the magnetic Laplacian result Theorem 2.1 and extending the Hardy-Littlewood-Pólya majorization technique to handle the union of a sequence and a shifted copy of the same sequence. We omit these proofs.

We chose to follow a more direct approach to proving Theorem 3.1, in the hope that it might help some future researcher to treat non-Dirichlet boundary conditions.

Acknowledgements. This work was partially supported by a grant from the Simons Foundation (\#204296 to Richard Laugesen), the National Science Foundation grant DMS-0803120 (Laugesen), and the Polish National Science Centre (NCN) grant 2012/07/B/ST1/03356 (Siudeja). We are grateful to the Banff International Research Station for funding our participation in the workshop on "Spectral Theory of Laplace and Schrödinger Operators" (July/August 2013), during which this paper was almost finalized.

\section{REFERENCES}

[1] Y. Aharonov and A. Casher, Ground state of a spin-1/2 charged particle in a two-dimensional magnetic field. Phys. Rev. A 19 (1979) 2461-2462.

[2] M.S. Ashbaugh and R.D. Benguria, Isoperimetric inequalities for eigenvalues of the Laplacian, Spectral theory and mathematical physics: a Festschrift in honor of Barry Simon's 60th birthday. In Vol. 76. Proc. of Sympos. Pure Math. Amer. Math. Soc. Providence, RI (2007) 105-139.

[3] C. Bandle, Isoperimetric Inequalities and Applications. In Vol. 7 of Monogr. Stud. Math. Pitman (Advanced Publishing Program), Boston, Mass. (1980).

[4] R.D. Benguria and H. Linde, Isoperimetric inequalities for eigenvalues of the LaPlace operator. Fourth Summer School in Analysis and Mathematical Physics. In Vol. 476 of Contemp. Math. Amer. Math. Soc. Providence, RI (2008) 1-40.

[5] V. Bonnaillie-Noël, M. Dauge, D. Martin and G. Vial, Computations of the first eigenpairs for the Schrödinger operator with magnetic field. Comput. Methods Appl. Mech. Engrg. 196 (2007) 3841-3858.

[6] L. Erdös, Rayleigh-type isoperimetric inequality with a homogeneous magnetic field. Calc. Var. Partial Differ. Eqs. 4 (1996) $283-292$.

[7] L. Erdös, Recent developments in quantum mechanics with magnetic fields, Spectral theory and mathematical physics: a Festschrift in honor of Barry Simon's 60th birthday. In Vol. 76, Part 1. Proc. of Sympos. Pure Math. Amer. Math. Soc. Providence, RI (2007) 401-428.

[8] L. Erdös, M. Loss and V. Vougalter, Diamagnetic behavior of sums of Dirichlet eigenvalues. Ann. Inst. Fourier, Grenoble 50 (2000) 891-907.

[9] S. Fournais and B. Helffer, Spectral Methods in Surface Superconductivity. In Vol. 77 of Progr. Nonlin. Differ. Eq. Appl. Birkhäuser Boston, Inc., Boston, MA (2010).

[10] R.L. Frank, A. Laptev and S. Molchanov, Eigenvalue estimates for magnetic Schrödinger operators in domains. Proc. Amer. Math. Soc. 136 (2008) 4245-4255.

[11] R.L. Frank, M. Loss and T. Weidl, Pólya's conjecture in the presence of a constant magnetic field. J. Eur. Math. Soc. (JEMS) 11 (2009) 1365-1383.

[12] B. Helffer and A. Morame, Magnetic bottles in connection with superconductivity. J. Funct. Anal. 185 (2001) $604-680$.

[13] A. Henrot, Extremum Problems for Eigenvalues of Elliptic Operators. Frontiers Math. Birkhäuser Verlag, Basel (2006).

[14] D. Henry, Perturbation of the Boundary in Boundary-value Problems of Partial Differential Equations. With editorial assistance from Jack Hale and Antônio Luiz Pereira. In Vol. 318 of London Math. Soc. Lect. Note Series. Cambridge University Press, Cambridge (2005).

[15] S. Kesavan, Symmetrization and Applications. In Vol. 3 of Series in Analysis. World Scientific Publishing Co., Hackensack, NJ (2006).

[16] A. Laptev and T. Weidl, Sharp Lieb-Thirring inequalities in high dimensions. Acta Math. 184 (2000) 87-111. 
[17] R.S. Laugesen, J. Liang and A. Roy, Sums of magnetic eigenvalues are maximal on rotationally symmetric domains. Ann. Henri Poincaré 13 (2012) 731-750.

[18] R.S. Laugesen and B.A. Siudeja, Sharp spectral bounds on starlike domains. J. Spectral Theory 4 (2014) 309-347.

[19] J.M. Luttinger, Generalized isoperimetric inequalities. J. Math. Phys. 14 (1973) 586-593, 1444-1447, $1448-1450$.

[20] NIST Digital Library of Mathematical Functions. Release 1.0.8 of 2014-04-25. Available at http://dlmf .nist.gov/.

[21] G. Pólya and G. Szegö, Isoperimetric Inequalities in Mathematical Physics. In Vol. 27 of Ann. Math. Stud. Princeton University Press, Princeton, N.J. (1951).

[22] D. Saint-James, Etude du champ critique $H_{c_{3}}$ dans une geometrie cylindrique. Phys. Lett. 15 (1965) $13-15$.

[23] S. Son, Spectral Problems on Triangles And Disks: Extremizers and Ground States. Ph.D. thesis, University of Illinois at Urbana-Champaign (2014). Available at https://www.ideals.illinois.edu/handle/2142/49349.

[24] J.W. Strutt (Lord Rayleigh), The Theory of Sound. In Vol. 1, 2nd edition. Macmillan and Co., London (1894). 